\title{
Multi-level Coupling of Dynamic Data-Driven Experimentation with Material Identification
}

\author{
John G. Michopoulos ${ }^{1}$ and Tomonari Furukawa ${ }^{2}$ \\ ${ }^{1}$ Computational Multiphysics Systems Laboratory \\ Special Projects Group, Code 6390.2 \\ Center for Computational Material Science \\ Naval Research Laboratory, USA \\ john.michopoulos@nrl.navy.mil \\ 2 ARC Centre of Excellence in Autonomous Systems \\ School of Mechanical and Manufacturing Engineering, J17, \\ The University of New South Wales, Sydney, NSW 2052, Australia \\ t.furukawa@unsw.edu. au
}

\begin{abstract}
We describe a dynamic data-driven methodology that is capable of simultaneously determining both the parameters of a constitutive model associated with the response of a composite material, and the optimum experimental design that leads to the corresponding material characterization. The optimum design of experiments may contain two parts. One involving the identification of the parameters that are tunable prior to performing the experiment such as specimen characteristics and a priori loading path. The other is involving the parameters characterizing the experiment during the experiment itself such as the directionality of the loading path for the case of multi-axial loading machine. A multilevel coupled design optimization methodology is developed and applied to demonstrate the concept. Essential to the process is the development of objective functions that express the quality of the experimental procedure in terms of the uniqueness and distinguishability associated with the inverse solution of the constitutive model determination. The examples provided are based on the determination of the linear constitutive response of a laminate composite material.
\end{abstract}

\section{Introduction}

The advent of dynamic data driven application systems (DDDAS) and associated technologies, as they have been evolving rapidly during the past five years [1] has stimulated us to explore various areas beyond the realm of how data can be used to dynamically drive computational model formation or drive the associated simulations. The opportunity to explore the feasibility of designing experiments -before and during the time of their execution- necessary for the collection of data needed for characterizing a material system, defines the focus and goal of this paper as a natural extension of our ongoing DDDAS efforts [2]3].

Utilization of data-driven design optimization practices to determine constitutive behavior parameters of materials under mechanical loadings has been 
traditionally based on experimental procedures with fixed architectures and no regard to how experimental designs may affect the quality of the material parameter estimation processes. However, the advent of multi-degree of freedom mechatronic systems capable of multidimensional mechanical loading 456], has introduced the potential of multiple designs for experimental processes to acquire the necessary behavioral data.

In the present paper we are proposing a multi-level design optimization methodology that intertwines three successive and dynamic design optimization subprocesses and is based to information theoretic grounds. One is responsible for the traditional material identification associated with the linear or nonlinear constitutive behavior; the other is responsible for the characterization of the proper loading path followed by a multidimensional loading frame online with the previous state of material characterization. The third is responsible for characterizing aspects of the experiment prior to conducting the experiment (i.e. offline). These aspects can be the geometrical characteristics of the shape of the specimen, lamination characteristics of it as well as the loading path itself as well.

Here we introduce the idea of a meta-objective functions that are constructed to express the performance of the constitutive model characterization optimization subprocess employed in the material identification level. Thus, the experimental design both in the offline and online senses, is generated dynamically as data are being acquired in a fashion that optimizes the performance of the optimization employed for the material parameter estimation.

The paper continues with a section that defines the proposed methodology. Subsequently, an application related to characterizing the elastic response of a composite material is described where the performance of the characterization process is defined in terms of the uniqueness and distinguishability of the obtained parameter set as solution of a singular value decomposition (SVD) problem. An application example is described and the paper concludes with a short discussion of the findings and the plans.

\section{Multi-level Approach}

The approach followed employs a process that involves multi-level design optimization that relates three stages or levels of intervention. The first stage involves the material model parameter characterization that occurs at has associated with it the master time marching loop. The second stage involves the determination of parameters that characterize the experiment during its exercise and therefore it is a real-time activity (online) relative to the experimental process intended for data acquisition. Its time marching loop is in parallel to that of the material identification one of stage one. The third stage is concerned with determining the parameters describing the experiment prior to actually performing the experiment. This stage offline stage has a time marching loop that is completely independent of the previous two. 


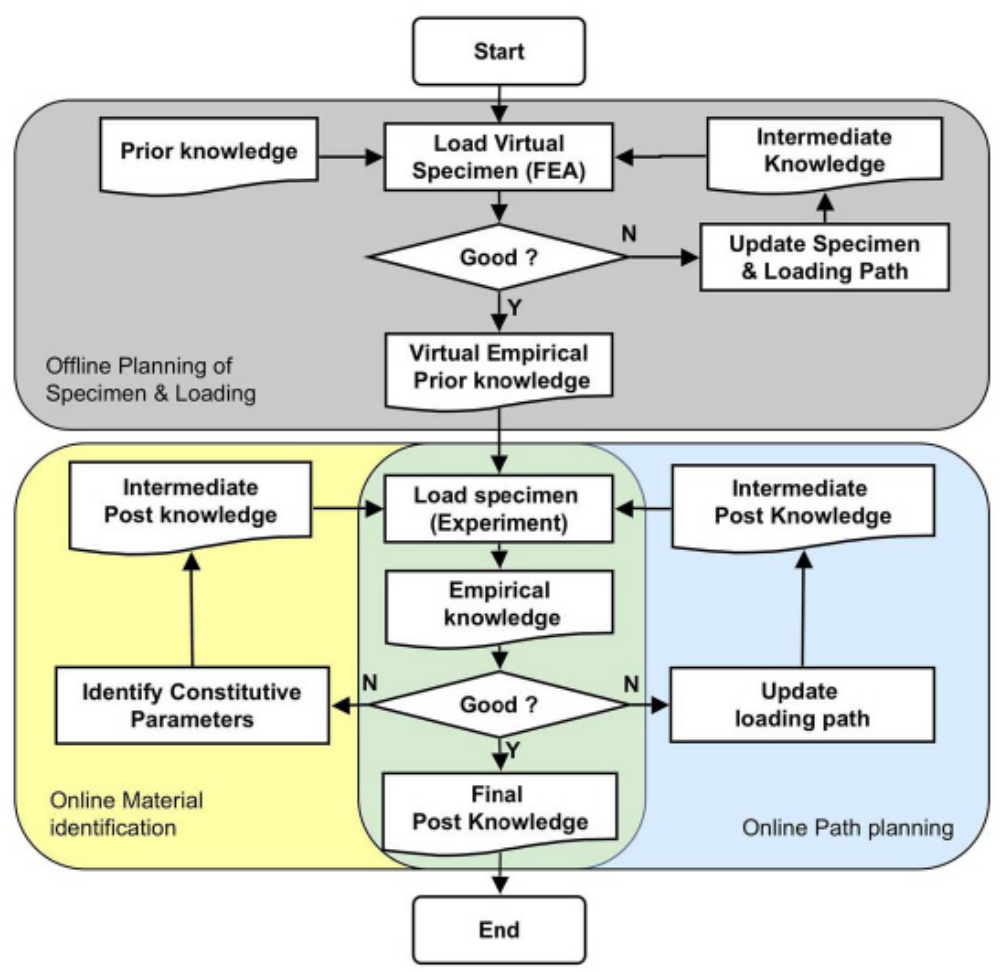

Fig. 1. Flow Chart of the interacting optimization levels or stages

A schematic representation of the linking of these stages is reflected by the block diagram in Fig. 1 where the flow chart of the interacting levels of design optimization are depicted.

As shown in Fig. 1 the online material identification (OMI) level contains the task of identifying the parameters associated with the behavior model in general, and the material constitutive model in particular. The branching node implies the existence of an optimizer that effectively implements the minimization of the difference between the behavior of the analytical model of the system that is under determination and the physical system itself as it is measured by the experimental frame tasked to expose it to the widest possible variety of excitation conditions. Then, a performance specification for the OMI is used to form the objectives of another optimizer in the level of the online path-planning level (OPP). The result of this optimization is the determination of the parameters describing the evolution of the loading path. Similarly, but separately in time, a performance specification for the OMI is used to form the objectives of the last optimizer in the level of the offline planning of specimen and loading (OPSL). From an information theoretic perspective levels OMI and OPP are using empirical knowledge derived from measured behavior of the respective systems and 
are producing final knowledge (identified behaviors) while they are building it iteratively trough successive stages of intermediate post knowledge. In the OPSL level prior knowledge based entirely on a priori considerations (like the initial geometric specification of the specimen) is used to feed the process and produce virtual empirical prior knowledge through various stages of intermediate knowledge.

\section{Composite Material System Application}

A linear anisotropic material with four moduli expressing its constitutive characteristics is considered as the system to be determined in level OMI. We have already demonstrated [78] that this problem can be reduced to the following linear (with respect to the unknown parameter vector) relation

$$
\mathbf{G}(\theta) \mathbf{q}_{M}=\mathbf{w}_{k},
$$

where $\mathbf{G}(\theta)=\left[\hat{\mathbf{g}}_{1}(\theta), \ldots, \hat{\mathbf{g}}_{n}(\theta)\right]^{T}$ is a $m \times 4$-dimensioned array that contributes the finite element approximation of the internal energy stored in the system from an increment of strain from point $K-1$ to point $K$ in a manner that does not contain the material moduli since that is contributed by the $4 \times 1$ array $\mathbf{q}_{M}$ of the unknown parameters on the left side of Eq. (11). In the right side the $m$-dimensioned array $\mathbf{w}_{K}$ contains the scaled external work that is applied as excitation into the system for all loading increments.

In Eq. (1) the right side represents the measured output of the system, while the left hand side represent the corresponding changing inside the system due to all possible excitation inputs. Its solution can be achieved by using any of the three methods available for implementation of least squares approximation 9 . Here we will focus on using SVD for the purpose of determining the parameters of the material model associated with level-OMI. We will also neglect additional computational performance criteria and focus only on potential measures of performance of the SVD implementation from an algorithmic perspective.

The solution containing the identified parameters according to Eq. (1) can be written in the form 778 .

$$
\mathbf{q}_{M}=\mathbf{G}(\theta)^{+} \mathbf{w}_{k}
$$

where

$$
\mathbf{G}(\theta)^{+}=\left\{\mathbf{G}(\theta)^{T} \mathbf{G}(\theta)\right\}^{-1} \mathbf{G}(\theta)^{T}
$$

is the pseudoinverse of $\mathbf{G}(\theta)$ and it exists uniquely only when $n \geq 4$ or when the system of linear equations represented by Eq. (2) is over-determined.

\section{Performance Measures}

What determines the quality of solving Eq. (1) is now reduced to determining the quality of applying Eq. (2) and therefore the quality of the process associated with establishing the pseudoinverse array defined by Eq. (3). 
We have identified $[6,7]$ that the concepts of "uniqueness" and "distinguishability" of the obtained solution, can be used as performance metrics for the quality of the determination of the parameter column array $\mathbf{q}_{M}$.

The uniqueness of the solution depends not only on the size of $\mathbf{G}(\theta)$ but also on whether $\mathbf{G}(\theta)^{T} \mathbf{G}(\theta)$ in Eq. (3) has and inverse matrix (or this matrix is fully ranked $(r=4))$. In order to guarantee the uniqueness and further prepare for designing optimal experiments via OPP or OPSL optimization, the proposed technique obtains the singular values of the matrix as a result of singular value decomposition (SVD) [10] as they express the factorization of in the form:

$$
\mathbf{G}(\theta)=\mathbf{U S V}^{T}
$$

where $\mathbf{U} \in \Re^{n \times n}$ and $\mathbf{V} \in \Re^{4 \times 4}$ are orthogonal to each other and $\mathbf{S} \in \Re^{n \times 4}$ is a diagonal array with real, non-negative singular values $s_{i}, \forall i \in\{1, \ldots, 4\}$. These singular values can be used to define the two measures characterizing parameter identification, one being the distinguishability and the other being the uniqueness of the solution and are described as follows.

Distinguishability can be defined as the property of the obtained solution to provide the largest possible variation of the measured response of two systems when their material parameters are very close to each other. It has been demonstrated that when two materials systems exhibit a small difference in their properties, then the difference of the values of their corresponding responses (observed experimentally) depend linearly on $S$ 10. Thus, any expression of the combined effect of the elements of $S$ as it increases, has the ability to distinguish two materials that are seemingly close to each other from a properties perspective, by producing exaggerated energy responses that are scaled values of these property variations.

We have defined distinguishability as the product of all singular values, and uniqueness as the inverse of the condition number of $\mathbf{G}(\theta)^{+}$according to

$$
F^{d} \equiv \prod_{i=1}^{n} s_{i} \quad \text { and } \quad F^{u} \equiv \frac{1}{c}=\frac{s_{\min }}{s_{\max }} \leq 1
$$

where $s_{\min }, s_{\max }$ are the minimum and maximum of the four singular values involved, respectively.

Uniqueness has been defined as the measure of whether $\mathbf{G}(\theta)^{T} \mathbf{G}(\theta)$ in Eq. (3) has an inverse matrix or not, is equivalent to the existence of $\left(\mathbf{S}^{T} \mathbf{S}\right)^{-1}$ as shown by substituting Eq. (4) into Eq. (3) that yields

$$
\mathbf{G}(\theta)^{+}=\mathbf{V}\left\{\mathbf{S}^{T} \mathbf{S}\right\}^{-1} \mathbf{S}^{T} \mathbf{U}^{T} .
$$

The necessary and sufficient condition for this to occur is that $\left|\mathbf{S}^{T} \mathbf{S}\right|=s_{1}^{2} s_{2}^{2} s_{3}^{2} s_{4}^{2} \neq$ 0 i.e., $s_{i} \neq 0, \forall i \in\{1, \ldots, 4\}$. It is important to underscore here that distinguishability increases as any of the $s_{i}$ increases, while uniqueness increases as the condition number decreases to unity. 


\section{Loading Path Optimization}

If in addition to determining the material parameters we require that this is achieved such as distinguishability and uniqueness are as high as possible, then we have defined the goals of the optimization involved in levels OPP and OPSL. The design variables at OPP level have to therefore be connected with what is controllable in an experimental setup used to acquire experimental data used for identifying the material parameters at OMI. Such parameters can be those that define the evolution of the loading path, such as total number of increments, loading path increment magnitude, and loading path increment orientation. For the case of a displacement controlled two degree of freedom (2-DoF) testing machine used for experimentation the parameter vector to be identified per loading increment could be could be formed by the measure of displacement increment and the angle denoting the change orientation of the loading path between to successive increments defined according to

$$
\Delta u_{K}=\left\|\mathbf{u}_{K+1}-\mathbf{u}_{K}\right\|, \quad \Lambda_{K, K+1}=\tan ^{-1}\left(\frac{u_{y \mid K+1}-u_{y \mid K}}{u_{x \mid K+1}-u_{x \mid K}}\right),
$$

with the total boundary displacement vector defined by its components along the two axes according to the usual definition $\mathbf{u}_{k} \equiv\left[u_{x}, u_{y}\right]_{k}=\left[u_{x \mid k}, u_{y \mid k}\right]$. Since increased uniqueness and distinguishability express a sense of reliability of the SVD process used for determining the material parameters in level OMI, we can define a vectorial objective function that needs to be maximized for maximum reliability and is formed from two components $J_{K, K+1}^{d}, J_{K, K+1}^{u}$ that represent the corresponding increments of distinguishability and uniqueness along an increment of loading [10. However, the solution of the two objective functions problem is not given by a single point but by a space satisfying the Paretooptimality, which is often referred to as Pareto-optimal front [10]. To avoid the computational cost associated with this approach the problem is reformulated in a manner that only one scalar objective function is constructed according to the following generalized form

$$
J_{K, K+1} \equiv(1-\mu) J_{K, K+K_{n}}^{d}+\mu J_{K, K+K_{n}}^{u} \rightarrow \max _{\Lambda_{K, K+K_{n}}}
$$

where each objective function is given by the scaled increment:

$$
J_{K, K+K_{n}}^{\alpha} \equiv \frac{\bar{F}_{0, K+K_{n}}^{\alpha}-F_{K}^{\alpha}}{F_{K}^{\alpha}}, \forall \alpha \in\{d, u\}
$$

and $\mu \in[0,1]$ is a weighting factor that controls the bias towards one or the other component of the objective function. The formulation expressed by Eqs. (819) avoids the computational tedious derivation of the Pareto-front. This scalar objective function can be extended beyond the algorithmic efficiency measures utilized up to now to capture computational efficiency metrics such as speed of computation. 


\section{Numerical Application}

To demonstrate the proposed concepts the material selected for generating the necessary simulated experimental data is a typical laminate constructed from an epoxy resin/fiber laminae system of type AS4/3506-1 with a balanced +/- 30 degrees stacking sequence.

All subsequent computational results have been produced by the implementation of the analysis presented earlier within MATLAB [11.

Figure 2 shows the resulting laminate material parameters evolution as a function of the number of the experimental points from the optimization conducted at level OMI.

The solution for the optimization at level OPP or OPSL is expressed in terms if the loading path defined on the $u_{x}-u_{y}$ plane as shown in Fig. 3.

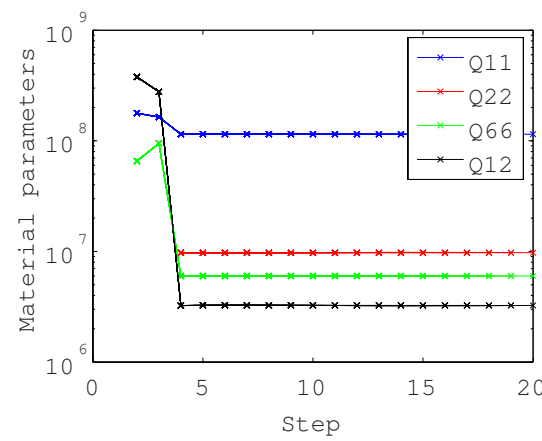

Fig. 2. Fig. 1 Evolution of determined material moduli from stage OMI

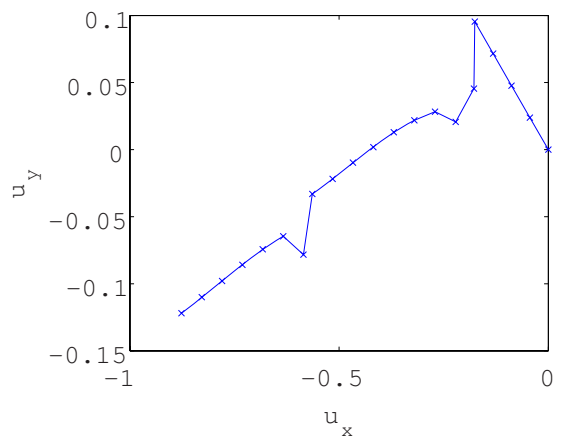

Fig. 3. Fig. 2 Evolution of loading path from stage OPP

\section{Conclusions}

The preliminary framework of a multi-level methodology was proposed; this methodology can succeed in both the determination of the parameters characterizing the response of a system, as well as characterizing the design parameters of an experiment required to collect data necessary for the systemic characterization.

The approach was applied in the context of an anisotropic material system. The systemic constitutive response of a linear anisotropic behavior to be identified was selected to be the elastic system fully defined from its five elastic moduli. These were the design variables for the OMI level of optimization. The experimental model considered for the OPP level of optimization was chosen to represent the description of a loading path in a 2-dimensional loading space. Implied here is the existence of a 2-degree of freedom loading frame, capable of applying such a loading path and measure both the path and reaction mechanical load characteristics for each increment. 
To achieve a definition of the objective function at OPP level the quantities of distinguishability and uniqueness were introduced as performance metrics of the design optimization process at level-2 that quantifies the performance of the SVD process employed. To achieve this, a two-component meta-objective function was constructed to be maximized. Maximization of this dual objective function leads to determines dynamically (for the OPP case) and in prior time (for the OPSL case) the experimental design specification in terms of loading path direction parameter.

Numerical simulation of the entire process was performed to demonstrate its feasibility. We demonstrated that the material moduli unknowns can be determined simultaneously to the loading path characteristics needed to design the necessary experiment.

Various extensions of this work will be attempted in the future, while simulation as the activity of exercising the determined model will also be added to complete the essential triad (dynamic and simultaneous physical model identification, design of experiments and design of simulation) of activities associated with a DDDAS [1].

Acknowledgement. The first author acknowledges the support by the National Science Foundation under grant 0540419 and the support of NRL's 6.1 corefunding program. Both authors acknowledge also the support by the Office of Naval Research.

\section{References}

1. Darema,F., Introduction to the ICCS2006 Workshop on Dynamic Data Driven Applications Systems. International Conference on Computational Science (3), (2006), 375-383

2. Michopoulos, J., Tsompanopoulou, P., Houstis, E., Farhat, C., Lesoinne, M., Rice, J., Joshi, A., On a Data Driven Environment for Multiphysics Applications, Fut. Generation Comp. Sys., 21(6), (2005), 953-968.

3. Farhat, C., Michopoulos, J. G., Chang, F. K., Guibas, L. J., Lew, A. J., Towards a Dynamic Data Driven System for Structural and Material Health Monitoring. International Conference on Computational Science, (3) (2006), 456-464.

4. Mast, P. W., Michopoulos, J. G., Thomas, R. W., Badaliance, R., and Wolock I., Characterization of strain-induced damage in composites based on the dissipated energy density: Part I - basic scheme and formulation. Int. Jnl. of Theor. and Applied Fract. Mech., 22, (1995), 71-96.

5. Michopoulos,J., Computational and Mechatronic Automation of Multiphysics Research for Structural and Material Systems, in "Recent advances in Composite Materials", Kluwer Academic publishing, (2003), 9-21.

6. Michopoulos, J., Mechatronically automated characterization of material constitutive respone. in: Proc. of the 6th World Congress on Computational Mechanics (WCCM-VI), (2004), 486-491.

7. Michopoulos, J.G., Furukawa, T., Kelly, D.W., A Continuum Approach for Identifying Elastic Moduli of Composites, Proceedings of the 16th European Conference of Fracture, Alexandroupolis, Greece, July 3-7, 2006 July 2006, Springer 2006. L, 1416 (2006). 
8. Michopoulos, J.G., Furukawa, T., Effect of Loading Path and Specimen Shape on Inverse Identification of Elastic Properties of Composites, Proceedings of IDETC/CIE 2006 ASME 2006, Paper DETC-2006-99724, CD-Rom ISBN: 0-79183784-X, (2006).

9. Lawson C.L., R.J. Hanson R.J., Solving Least Squares Problems, Prentice-Hall, Englewood Cliffs, NJ, (1974). Reprinted by SIAM Publications, Philadelphia, PA, (1996).

10. Michopoulos, J.G., Furukawa, T., Design of Multiaxial Tests for Characterizing Anisotropic Materials, Int. J. Numer. Meth. Engng., In print.

11. The Mathworks, Matlab, http://www.mathworks.com 